\title{
Anti Tumor Necrosis Factor Alpha and Renal Disease in Rheumatoid Arthritis
}

\author{
Oriba Dan Langoya ${ }^{\top}$ \\ 1 Makerere University \\ Funding: The author(s) received no specific funding for this work. \\ Potential competing interests: The author(s) declared that no potential competing interests exist.
}

\begin{abstract}
Tumor necrosis factor alpha (TNF- $\alpha)$ is a potent and critical proinflammatory cytokine produced by the immune cells especially macrophages and T lymphocytes, however other cell of the immune system such as white blood cells, renal tubular cells, vascular endothelial cells also produce it in small quantities. TNF- $\alpha$ has both physiological and pathological roles, an important family of both the soluble and cell bound subunit of cytokines and its primary role is promotion of inflammation. It is also involved in lymphoid development and has cytotoxic and apoptotic effects. This master proinflammatory cytokine has been implicated in diseases such as rheumatoid arthritis (RA) especially with renal involvement, and others include ankylosing spondylitis, Crohn's disease. The advent of anti TNF- $\alpha$ has also promising results in managing these conditions. Epidemiologically we know that renal insufficiency is a common occurrence in patients with rheumatic arthritis and this limits the use of non-steroidal anti-inflammatory drugs (NSAIDs) and disease modifying anti-rheumatic drugs (DMARDs). The buildup of TNF- $\alpha$ levels in chronic kidney disease may justify the use of these agent.
\end{abstract}

Introduction

Renal insufficiency is a common comorbidity in patients with inflammatory and autoimmune diseases such as RA, psoriasis and TNF- $\alpha$ a pleiotropic cytokine produced mainly by activated macrophages/monocytes and most often associated with proinflammatory properties, plays a pivotal role in the inflammatory cascade leading in the pathogenesis of renal injury. Decreased renal function is a limiting factor for the use of NSAIDs and DMARDs. Therefore, availability of therapeutic options like anti TNF- $\alpha$ would improve treatment outcomes in these patients ${ }^{1-2}$. From an etiological point, TNF- $\alpha$ is directly involved in renal injury due its local production by stimulated intrinsic renal cells which seems to be functionally more relevant in renal inflammation ${ }^{3}$ and chronic kidney disease (CKD) constitute a chronic inflammatory state, as demonstrated by elevation of various growth factors, inflammatory mediators, and inflammatory markers, such as C-reactive protein (CRP), interleukin-6, tumor necrosis factor alpha (TNF- $\alpha$ ), or monocyte chemoattractant protein-1 concentration 4. In addition, glomerular endothelial cells are a target of TNF- $\alpha$, which then amplifies the production of 
complement cascade by endothelial cells and this mechanism plays a critical role in the pathogenesis of murine immune complex-mediated glomerulonephritis ${ }^{3}$. This therefore justifies the importance anti TNF- $\alpha$ drugs in stabilizing renal function in RA patients with CKD and or secondary renal amyloidosis by suppression of inflammation 4 .

\section{Mechanism of action of anti TNF- $\alpha$ in renal disease}

Generally, anti TNF- $\alpha$ exerts its effect by suppression of proinflammatory state manifested by elevated levels of growth factors, inflammatory mediators and markers, like C-reactive protein (CRP), interleukin- 6 and the potent cytokine TNF- $\alpha$ in CKD associated with RA.

\section{Anti TNF- $\alpha$ and Renal Disease Review}

With activated macrophages being the principle source of TNF- $\alpha$, it is not therefore unusual that renal TNF expression increases with leukocyte infiltration in renal diseases, which therefore potentiates glomerular injury ${ }^{5}$. Garrouste et al., evaluated patients treated with anti TNF- $\alpha$ for CKD and transplant recipients, and found that complete response occurred in about $56 \%$ of patients and $40 \%$ in patients with chronic rheumatic disease. However, the major complications associated with treatment with anti-TNF- $\alpha$ were serious infections in about $50 \%$ of patients. The major agents implicated was bacterial in etiology however reaction of varicella Zoster and CMV and fungal infections were noted in some patients 6

Patients RA with concurrent CKD face challenges in management as the mechanism of Kidney dysfunctions can be due to the direct toxic effects of NSAIDS and DMARDS. However as earlier mentioned, the chronic systemic inflammatory state well mediated by TNF- $\alpha$ build-up contribute to progressive loss of renal function in these patients. In a study by Kim et al., administration of anti TNF- $\alpha$ resulted in stabilization of renal functions in patients with RA and CKD and this was mediated by the anti-inflammatory role of these drugs (etanercept, infliximab, and adalimumab) which undergoes hydrolysis in lysosomes and therefore, have no adverse effects on renal functions compared to NSAIDs and DMARDs with significant nephrotoxicity limiting their use in patients with renal impairment ${ }^{4}$.

This demonstrates the therapeutic benefit of selective blockade of TNF- $\alpha$ in improving renal function by attenuating renal inflammation. However there is a caveat to this beneficial effect of anti TNF- $\alpha$ as reported by Stokes et al., who acknowledged that patients with RA on anti TNF- $\alpha$ may develop glomerulonephritis (GN) through induction of RA related nephropathy or denovo autoimmune disorders and this was justified by the presence and formation of antinuclear antibodies (ANA), anti-double stranded DNA (dsDNA) anti- bodies, and anticardiolipin antibodies (ACL) in a significant subset of RA patients, which seemed to be mediated by binding of anti-TNF- alpha on immune cells with propensity to induce release of immunogenic nucleosomal antigens, therefore suggesting a role for TNF- $\alpha$ in normal immune regulation 7-8

In another review, Ernandez \& Mayadas, alluded to the kidney as the organ frequently involved in inflammatory and autoimmune conditions. Notably emphasis is currently placed on TNF- $\alpha$ as a major pathway for pathogenesis of renal injury through it proinflammatory and immunosuppressive role especially in lupus nephritis. In affirming this statement, the development of anti TNF- $\alpha$ has shown promising results in anti-neutrophil cytoplasmic antibody (ANCA)-associated vasculitis nephritis ${ }^{3}$. However, in another case series report by Premužić et al., the development of IgA nephropathy 
although a rare complication of anti TNF- $\alpha$ was documented in patients with RA and Diabetes but without prior chronic renal injury. Subsequent discontinuation of anti TNF- $\alpha$ with institution of immunosuppressive therapy was associated with improvements in renal function ${ }^{8}$. This supports the role of anti TNF- $\alpha$ in induction of autoimmunity. To add some weight to the latter statement, Stokes et al., reported an etiologic role for anti TNF- $\alpha$ therapy in the initiation of GN and this view gained support from the temporal association of this drug use with new-onset nephropathy, which often was accompanied by new serologic abnormalities, in individuals who had no prior evidence of renal disease ${ }^{7}$. Another study also pointed out TNF- $\alpha$ as instrumental pathogenesis in the development of renal dysfunction and cell death in response to sepsis or ischemia-reperfusion injury by induction of renal cell apoptosis ${ }^{9}$. Notably, inhibition of TNF transcription or selective inhibition of TNF synthesis is an effective anti-TNF strategy in the management of this condition ${ }^{9}$. In addition, anti TNF- $\alpha$ was well tolerated and found to be safe in patients with renal involvement of amyloid $\mathrm{A}$ amyloidosis ${ }^{1}$.

\section{Conclusion}

Anti TNF- $\alpha$ agents appears to be safe and well tolerated in patients with RA and CKD, presenting a novel alternative to patients with contraindications to NSAIDS and DMARDS. However, surveillance for bacterial, viral reactivation and for much rare complications of treatment with this agent should involve careful surveillance for proliferative lupus GN, pauciimmune necrotizing, crescentic GN, membranous GN and renal vasculitis.

\section{References}

1. Hueber AJ, Tunc A, Schett G, Manger B. Anti-tumour necrosis factor alpha therapy in patients with impaired renal function. Ann Rheum Dis. 2007;66(7):981-982. doi:10.1136/ard.2006.069211

2. Ernandez T, Mayadas T. Immunoregulatory role of TNF $\alpha$ in inflammatory kidney diseases. Kidney Int. 2009;76(3):262276. doi:10.1038/ki.2009.142 3. Ernandez T, Mayadas T. Immunoregulatory role of TNF $\alpha$ in inflammatory kidney diseases. Kidney Int. 2009;76(3):262-276. doi:10.1038/ki.2009.142 4. Kim HW, Lee CK, Cha HS, Choe JY, Park EJ, Kim J. Effect of anti-tumor necrosis factor alpha treatment of rheumatoid arthritis and chronic kidney disease. Rheumatol Int. 2015;35(4):727-734. doi:10.1007/s00296-014-3146-4

5. Vielhauer V, Mayadas TN. Functions of TNF and its Receptors in Renal Disease: Distinct Roles in Inflammatory Tissue Injury and Immune Regulation. Semin Nephrol. 2007;27(3):286-308. doi:10.1016/j.semnephrol.2007.02.004

6. Garrouste C, Anglicheau D, Kamar N, et al. Anti-TNF $\alpha$ therapy for chronic inflammatory disease in kidney transplant recipients Clinical outcomes. Med (United States). 2016;95(41). doi:10.1097/MD.0000000000005108

7. Stokes MB, Foster K, Markowitz GS, et al. Development of glomerulonephritis during anti-TNF-alpha; therapy for rheumatoid arthritis. Nephrol Dial Transplant. 2005;20(7):1400-1406. doi:10.1093/ndt/gfh832

8. Premužić V, Padjen I, Cerovec M, Ćorić M, Jelaković B, Anić B. The Association of TNF-Alpha Inhibitors and Development of IgA Nephropathy in Patients with Rheumatoid Arthrit is and Diabetes. Case Reports Nephrol . 2020;2020:18. doi:10.1155/2020/9480860

9. Donnahoo KK, Shames BD, Harken AH, Meldrum DR. The role of tumor necrosis factor in renal ischemia reperfusion injury. doi:10.1097/000053921999 07000J Urol 00068 . 1999;162(1):196203. 
